\title{
ESTUDO DA VARIABILIDADE DA COLUNA DE ÁGUA PRECIPITÁVEL (PWC) NO SEMIÁRIDO BRASILEIRO PARA CALIBRAÇÃO DE FOTÔMETRO SOLAR
}

\author{
J. G. CERQUEIRA JUNIOR ${ }^{1,2}$, J. H. FERNANDEZ ${ }^{1}$, J. J. HOELZEMANN ${ }^{1}$, N. M. P. LEME $^{1}$ e C. T. SOUSA ${ }^{2}$ \\ ${ }^{1}$ Universidade Federal do Rio Grande do Norte \\ ${ }^{2}$ Instituto Nacional de Pesquisas Espaciais - INPE \\ jgcjunior@gmail.com
}

Artigo submetido em abril/2014 e aceito em agosto/2014

DOI: $10.15628 /$ holos.2014.2065

\begin{abstract}
RESUMO
A água está presente na atmosfera na forma de vapor, desde o nível do solo até a estratosfera. Como principal componente do ciclo hidrológico, a variação em sua quantidade sobre uma região provoca alterações nas condições climáticas, afetando assim a situação econômica e as condições de vida da população local. O fotômetro solar permite monitorar a variação dessa coluna de vapor de água, ou coluna de água precipitável (PWC), utilizando a radiação solar na banda de $940 \mathrm{~nm}$, que sofre uma forte absorção ao atravessar a atmosfera. Para a calibração do fotômetro solar desenvolvido nesse trabalho, foi utilizado um sítio na região semiárida do
\end{abstract}

estado do Rio Grande do Norte, próximo à cidade de Caicó. Foi aplicado o Método de Langley Modificado (MLM) que é uma variação do Método de Langley. Essa metodologia permite calibrar o fotômetro solar em campo aberto, que sob a condição de estabilidade óptica da atmosfera, permite obter-se constantes de calibração com melhores índices de incerteza que em laboratório. Os resultados, durante o período estudado, mostraram que não ocorreu a esperada estabilidade óptica da atmosfera para permitir a calibração do equipamento.

\section{VARIABILITY STUDY OF THE PRECIPITABLE WATER COLUMN (PWC) IN SEMI-ARID BRAZILIAN REGION FOR SUN PHOTOMETER CALIBRATION}

\begin{abstract}
Water is present in the atmosphere as vapor, from ground level to stratosphere. As a major component of the hydrological cycle, water quantity variation over a region causes climate change, affecting the economic and living conditions of the local population. The sun photometer allows you to monitor the variation of this precipitable water column (PWC) i.e. water vapor in atmosphere using solar radiation in the $940 \mathrm{~nm}$ band, where strong absorption occurs. For calibration of the sun photometer developed in this work, we investigated
\end{abstract}

a site in the semiarid region of the Rio Grande do Norte state, near the city of Caicó, applying Modified Langley Method (MLM) which is a variation of the Langley Method. This methodology allows us to calibrate sun photometer for PWC in the open field, which under atmosphere optical stability condition makes possible to obtain best calibration constants than in laboratory. The results, during the study period, showed that the expected atmosphere optical stability for the calibration of the equipment did not occur.

KEYWORDS: Aerosol Optical Depth, Sun Photometer, Calibration, Modified Langley Method. 


\section{INTRODUÇÃO}

O vapor de água é um importante componente do processo hidrológico, estando, intrinsecamente ligado à climatologia global. É o constituinte mais variável da atmosfera e também contribui para o efeito estufa. Afeta diretamente o sistema climático global ao absorver o fluxo de calor sensível ou liberar o fluxo de calor latente com a mudança de estado da água [12]. O vapor de água afeta a energia atmosférica por meio das interações radiativas, um fato de alta relevância para as comunicações e aplicações em sensoriamento remoto [19]. O vapor de água está entre os componentes atmosféricos que apresentam maior interesse para as ciências atmosféricas, meteorológicas, hidrológicas e de pesquisas climáticas. A radiação solar adiciona energia à atmosfera terrestre pela absorção direta, e de forma indireta pela absorção da radiação térmica transmitida da superfície do planeta, criando assim o movimento convectivo na troposfera.

O vapor de água produz uma grande realimentação negativa no esfriamento radiativo da superfície [8]. Dentre todos os elementos que colaboram com o efeito estufa o vapor de água tem papel de destaque, por ser o maior constituinte na troposfera depois do nitrogênio e oxigênio, por conseguinte o que mais contribui para o efeito estufa [25]. O vapor de água é um parâmetro chave para as análises dos sistemas climáticos (gases do efeito estufa), em particular sobre altas latitudes onde mostra uma importante variabilidade sazonal [2]. O efeito estufa é necessário para gerar o aquecimento atmosférico, indispensável à sobrevivência dos seres vivos. Entretanto pode tornarse danoso a partir da elevação progressiva das concentrações na Terra de alguns gases que contribuem para a elevação deste efeito $(\mathrm{CO} 2, \mathrm{CH} 4$, etc.) e que podem produzir um aumento na temperatura da atmosfera, com consequências catastróficas para a biosfera.

Os padrões de precipitação estão intimamente ligados à umidade na atmosfera, assim como a evaporação, condensação e processos de transporte. Os esparsos dados não permitem boas estimativas da condensação e do transporte vertical do vapor de água, que podem ser frequentemente dominada por processos convectivos em baixa escala, tornando difícil sua avaliação de maneira global [16]. A coluna de água precipitável (PWC) na atmosfera terrestre é dada pela medida desta coluna, dada em centímetros, desde a superfície do planeta até a pressão atmosférica de $50 \mathrm{hPa}$, aproximadamente $20.000 \mathrm{~m}$, observado em sensores de umidade instalado em balões meteorológicos.

Uma maneira de medir o vapor de forma pontual é usando-se um sensor de umidade relativa, instalado ao nível do solo ou embarcado em balão meteorológico. Assim o sensor irá quantificar o valor percentual de água em relação a uma porção gasosa da atmosfera. O fotômetro, valendo-se de um algoritmo de cálculo matemático estima a atenuação ou extinção (absorção e/ou espalhamento) da radiação solar, quantificando assim o vapor de água integrado de toda a porção de água desde o topo da atmosfera até a superfície onde se encontra o fotômetro.

\section{REVISÃO TEÓRICA}

\subsection{A Lei de Beer}

A lei de Bouguer-Lambert-Beer ou simplesmente lei de Beer descreve a variação da radiância espectral $d V(\lambda)$ dada em Watts.área.esfero-rad.frequência $\left(W \cdot m^{-2} \cdot s r^{-1} \cdot \mu m^{-1}\right)$, causando 
extinção da radiação incidente $V_{o}(\lambda)$ ao longo de um caminho óptico $d(s)$ devido a presença de partículas (átomos, moléculas e aerossóis) que absorvem ou espalham a radiação solar. A Figura 2.1 ilustra o cenário da aplicação da lei para a extinção da radiação solar ao passar pela atmosfera terrestre, sofrendo espalhamento ou absorção. A intensidade da radiação monocromática $V_{\lambda}$, torna-se $V_{\lambda}+d V_{\lambda}$ depois de atravessar $d S$. Nesse trabalho a dedução da lei de Beer será abordada em termos de irradiância solar, que é a densidade de fluxo radiante, dada em Watts por área (W. $\left.\mathrm{m}^{2}\right)$.

Então:

$$
d V_{\lambda}=-k_{\lambda} \rho V_{\lambda} d S
$$

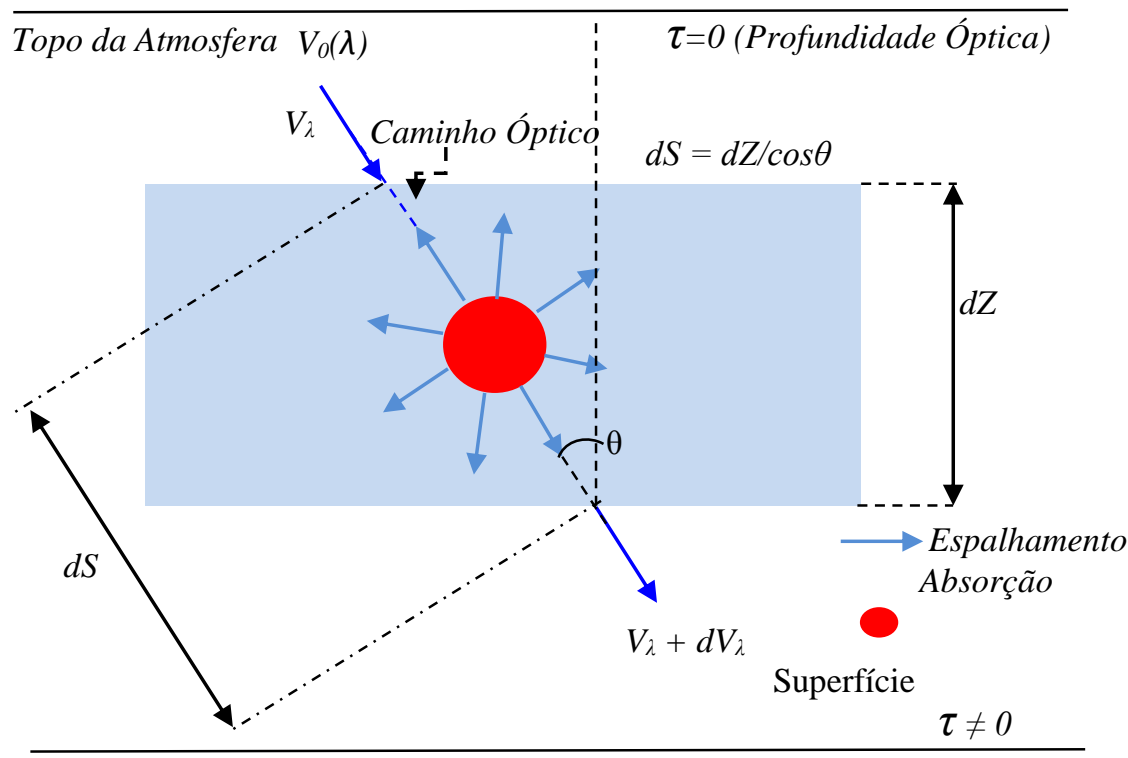

Figura 2.1. Representação esquemática da atenuação da irradiância solar incidente.(Adaptado de [4])

Obs.: O sinal negativo é devido à extinção, ou seja, diminui-se a intensidade da radiação.

Onde: $\rho=$ densidade do meio ; $k_{\lambda}=$ seção reta de choque da massa que causa a extinção (absorção + espalhamento) da radiação " $\lambda$ ” (área/massa).

Da Equação 2.1 temos:

$$
\int_{V_{\lambda 0}}^{V_{\lambda}} \frac{d V_{\lambda}^{\prime}}{V_{\lambda}^{\prime}}=\frac{1}{\cos \theta} \int_{0}^{Z}-k_{\lambda} \rho d Z,
$$

Onde $V^{\prime}{ }_{\lambda}$ foi definido para diferenciar o integrando. Fazendo a intensidade de $S=0$, teremos $V_{\lambda}=0$. Assim a intensidade emergente a uma distância " $S$ " ao longo de $d S$ pode ser obtida integrando a Equação 2.2.

Onde $V^{\prime}{ }_{\lambda}$ foi definido para diferenciar o integrando, assim, integrando o lado esquerdo: 


$$
\left.\ln V_{\lambda}^{\prime}\right|_{V_{\lambda 0}} ^{V_{\lambda}}=-\frac{k_{\lambda}}{\cos \theta} \int_{0}^{Z} \rho d Z,
$$

Substituindo os limites da integração:

$$
\ln V_{\lambda}-\ln V_{\lambda 0}=-\frac{k_{\lambda}}{\cos \theta} \int_{0}^{Z} \rho d Z
$$

Usando-se as propriedades do In têm-se:

$$
\ln \frac{V_{\lambda}}{V_{\lambda 0}}=-\frac{k_{\lambda}}{\cos \theta} \int_{0}^{z} \rho d Z,
$$

Fazendo $\tau_{\text {Total }}=-k_{\lambda} u$ (profundidade óptica total) onde, $u=-k_{\lambda} \int_{0}^{z} \rho d Z$ e $m=\frac{1}{\cos \theta}$ (massa óptica). Assim a lei de Beer é mostrada na Equação 2.6.

$$
V_{\lambda}=V_{10} e^{-\frac{k_{\lambda}}{\cos \theta} \int_{0}^{z} \rho d z}
$$

Incluindo uma compensação com relação à distância terra/sol, que varia anualmente com o quadrado da distância devido à órbita elíptica da terra. Logo a lei de Beer pode ser escrita como na Equação 2.7 [11]. Onde $D_{\text {Atual }}$ é a distância terra-sol no dia da medida e $D_{\text {Min }}$ é a menor distância entre terra-sol.

$$
V_{\lambda}=V_{\lambda O}\left(\frac{D_{\text {Atual }}}{D_{\text {Min }}}\right)^{2} e^{-\tau_{\text {total }} \cdot m} .
$$

A profundidade óptica total " $\tau_{\text {total }}$ " é o fator de extinção da radiação solar ao passar pela profundidade $d Z$ (trajeto $d S$ ) e é dada pelo somatório de fatores como: espalhamento Rayleigh, absorção e espalhamento por aerossóis, camada de ozônio, gases traço $\left(\mathrm{CO}_{2}, \mathrm{CH}_{4}, \mathrm{SO}_{2}\right.$, etc. $)$ e vapor de água como mostrada na Equação 2.8 [5].

$$
\tau_{\text {total }}=\tau_{\text {Ray }}+\tau_{\text {Aer }}+\tau_{O_{3}}+\tau_{\text {gas }} .
$$




\section{MATERIAIS E MÉTODOS}

\subsection{MÉTODO DE LANGLEY MODIFICADO}

\subsubsection{Introdução}

O método de Langley Modificado (MLM) é uma variação do método de Langley (ML), e são normalmente aplicados em sítios com alta altitude, sendo o mais conhecido o observatório no monte MAUNA LOA/Hawaii/USA que fica a $3400 \mathrm{~m}$ acima do nível do mar. Nesse sítio o GSFC (Goddard Space Flight Center) que pertence a NASA (National Aeronautics and Space Administration) calibra o fotômetro solar de referência da rede AERONET. Essa metodologia é utilizada na calibração de fotômetro solar, sendo utilizada para a calibração de equipamentos que operam desde a faixa UV até o Infravermelho próximo, para medição da AOD (Profundidade Óptica por Aerossóis) usando o método de Langley.

Geralmente, somente sítios em grandes altitudes propiciam alta estabilidade óptica durante as medições, fator fundamental para a aplicação da metodologia. No entanto, em sítio de média altitude situado em Boulder, Colorado, reportou sucesso na aplicação do método de Langley em alguns resultados [7]. Recentemente foi mostrado que os locais de baixa altitude, distantes dos centros urbanos e industriais podem proporcionar uma profundidade óptica estável, similar a altas altitudes [12].

A transmitância do vapor de água na atmosfera não segue as mesmas leis de atenuação da radiação solar na atmosfera gerada por aerossóis e outros gases. Para estimar a quantidade de vapor de água presente na atmosfera com um fotômetro solar, considera-se a banda de 940nm por esta apresentar uma forte absorção. Foi em 1974 que ocorreu a primeira aplicação do que viria a se chamar Método Modificado de Langley (MLM) [27], utilizando a dependência da transmitância da atmosfera pela raiz quadrada, para a banda de 940 $\mathrm{nm}$. Essa mesma dependência foi aplicada na banda de 940nm, onde mediu-se a variação da irradiância nessa banda [17]. Introduzindo uma modificação no método de Langley, [18], usando radiômetros em 940nm e na banda adjacente de 870nm e com medidas simultâneas, foi determinada a quantidade de água precipitável na atmosfera. A transmitância da água $T_{w}$ inclui termo de decaimento exponencial da radiação solar no MLM, devido à coluna de vapor de água $(P W C)$ na atmosfera, que em primeiro lugar foi calculado usando o modelo de [26].

Um aprimoramento do MLM foi proposto em [6, 3], tornando o método mais preciso, permitindo assim o uso de modelos atmosféricos na metodologia de estimativa do PWC. Um trabalho comparativo entre medida de água precipitável por fotômetro solar e radiossondas, aplicando o MLM [6]. A evolução do MLM veio com o desenvolvimento de modelos de transmitância atmosférica que ano a ano apresentavam melhorias em suas resoluções. $O$ primeiro procedimento de calibração V1 do fotômetro AERONET/NASA usava o LOWTRAN (Low spectral resolution transmitance model) para o cálculo das constantes " $a$ " e " $b$ ", desenvolvido por [10]. Esse modelo foi aplicado para a estimativa do PWC [2]. No trabalho apresentado em 1995 [13], que trata da calibração na banda de $940 \mathrm{~nm}$, considerou-se o MODTRAN2 (Moderate spectral resolution transmitance model-2), [1]. A resolução do modelo MODTRAN, quando comparada com o LOWTRAN, teve um incremento, passando de $50 \mathrm{~nm}$ para $5 \mathrm{~nm}$ de resolução espectral, permitindo assim uma melhor caracterização das faixas de absorção ou espalhamento. 
Em 1996 um comparativo entre os modelos de estimativa da transmitância atmosférica LOWTRAN7, MODTRAN3, FASCOD3P (Fast atmospheric signature code) e o modelo de Thomason [48], foi feito um estudo da transmitância atmosférica para a banda de $940 \mathrm{~nm}$. Nesse trabalho foram feitos comparativos entre vários tipos de equipamentos que medem de maneira distinta o vapor de água (Radiômetro de micro-ondas- MWR; fotômetro solar e radiossondas). Os modelos mostram valores em $\mathrm{g} / \mathrm{cm}^{2}$, ou seja, $1 \mathrm{~g} / \mathrm{cm}^{2}$ é igual a $1 \mathrm{~cm}$ de coluna líquida de água, onde $1 \mathrm{~cm}$ $\left(\mathrm{H}_{2} \mathrm{O}\right)=3.345 \times 10^{23}$ moléculas $/ \mathrm{cm}^{2}[19]$.

\subsubsection{Gerando a reta do MLM}

A variação da intensidade da atenuação (absorção ou espalhamento), dada pela lei de Bouguer-Lambert-Beer com modificação para a banda de $940 \mathrm{~nm}$ [3], é mostrada na Equação 3.1. $O$ incremento do termo $T_{w}$, mostrado na Equação 3.2, ajusta a variação da transmitância do PWC na atmosfera:

$$
\begin{gathered}
V_{(940)}=V_{o(940)}\left(\frac{D_{\text {Atual }}}{D_{\text {min }}}\right)^{2} e^{-\left(\tau_{A}+\tau_{R}\right) \cdot m} \cdot T_{w}, \\
T_{w}=e^{-\left(a w^{b} m^{b}\right)} .
\end{gathered}
$$

As constantes " $a$ " e " $b$ ", mostradas na Equação 2.19, são estimadas usando-se modelo de transmitância atmosférica $[8,5,2]$. Os valores de " $a$ " e " $b$ " para os filtros adquiridos da ANDOVER CORPORATION, na faixa de 940nm para uso no FSM-4 são mostrados na Tabela 3.1.

Tabela 3.1. Valores das constantes a e b para os filtros de $940 \mathrm{~nm}$.

\begin{tabular}{|c|c|c|c|}
\hline $\begin{array}{c}\text { Tipo filtro - } \\
\text { 940FS10-12.5 }\end{array}$ & Freq. Central & Cte. “ $\boldsymbol{a}$ " & Cte. “ $\boldsymbol{b}$ " \\
\hline S/N S153-01 & $939.64 \mathrm{~nm}$ & 0.69 & 0.57 \\
\hline S/N S153-02 & $939.58 \mathrm{~nm}$ & 0.69 & 0.57 \\
\hline
\end{tabular}

A linearização da Equação 3.1, aplicando-se o logaritmo natural, é mostrada na Equação 3.3, gerando assim o MLM.

$$
\ln V_{940}-2 \ln \left(\frac{D_{\text {Atual }}}{D_{\text {min }}}\right)+\left(\tau_{A}+\tau_{R}\right) m=\ln V_{o}-a W^{b} m^{b},
$$

Assim tem-se: $W=$ coluna integrada de vapor de água; $m=$ =massa óptica; "a" e "b", constantes intrínsecas do filtro devido sua banda passante.

Onde na Equação da reta:

$$
\begin{gathered}
y=b-a x \\
y=\ln V_{\lambda(940)}-2 \ln \left(\frac{D_{\text {Atual }}}{D_{\text {min }}}\right)+\left(\tau_{A}+\tau_{R}\right) m .
\end{gathered}
$$

Onde :

$$
\mathrm{b}=\ln V_{0}
$$




$$
\begin{gathered}
x=m^{b} \\
a=a W^{b}
\end{gathered}
$$

Gerando o gráfico de $\ln V_{\lambda(940)}-2 \ln \left(\frac{D_{\text {Atual }}}{D_{\text {min }}}\right)+\left(\tau_{A}+\tau_{R}\right) m \times m^{b}$.

A constante extraterrestre do equipamento ou coeficiente de calibração $V_{0}$, é encontrada para $m^{b}=0$ após retas ajustadas, quando então é analisado o desvio padrão amostral desta constante. É geralmente aceito que, se os valores de $V_{0}$ se repetem em vários dias de medições independentes, eles podem ser considerados como confiáveis [21]. Para obtenção da constante de calibração $V_{0}$ para o MLM, os pontos para ajuste da reta devem ser coletados ao menos durante um período de 1.5 - 2.0 horas [20]. O coeficiente de variação de $V_{0}$ no fotômetro AERONET no Observatório Mauna Loa obtido durante várias seções de calibração ficam entre cerca dê 0,25 a $0,50 \%$, bandas espectrais localizadas no espectro visível e infravermelho próximo. Para a banda de 940 a variação do $V_{0}$ e entre aproximadamente entre $1 \%$ a $3 \%$.

Em sítio com altitude de apenas $95 \mathrm{~m}$ foi obtida a calibração de um equipamento MFRSR (Multi-Filter Rotating Shadowband Radiometer), que utiliza o MLM, foram realizadas medidas in situ em Xiang He (39॰47'54"N, 116॰57'28”E) [12]. O experimento foi feito em condições de céu claro, com massa de ar $(\mathrm{m})$ entre 2 e 6 , obtendo coeficiente de determinação próximo de 1 , indicando atmosfera estável no período das medidas.

\subsubsection{Calculando a coluna de PWC}

Colocando a Equação 3.3 em função de "W", [20,22,2,12] têm-se a coluna de vapor de água integrada, que é calculada pelo esquema da Figura 3.2.

Onde PWC, que é dada em centímetros (cm), é obtida a partir da Equação 3.4, ou seja, integra os valores na coluna atmosférica da superfície até o topo da atmosfera.

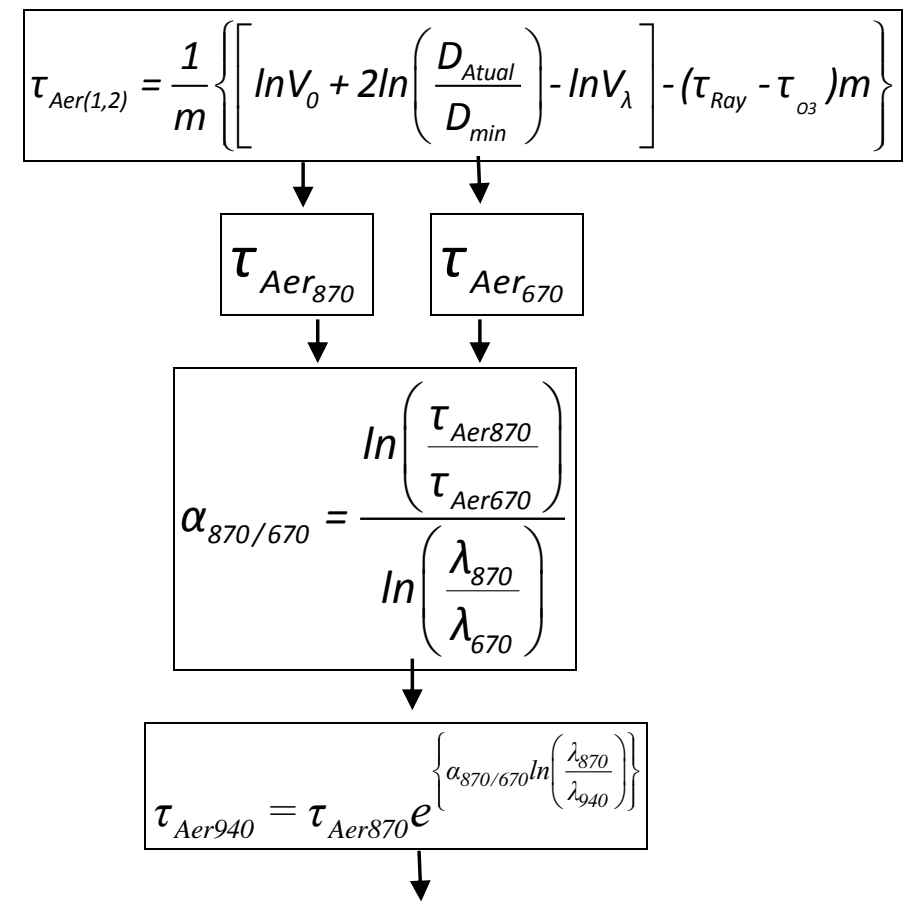




$$
P W C=\frac{1}{m}\left\{\frac{1}{a}\left[\ln V_{0(940)}+2 \ln \left(\frac{D_{\text {atual }}}{D_{\min }}\right)-\ln V_{940}-\left(\tau_{\text {Ray }(940)}+\tau_{\text {Aer(940) }}\right) m\right]\right\}^{\frac{1}{b}}
$$

Figura 3.2. Diagrama da sequência de cálculo do PWC.

\subsection{DESENVOLVIMENTO DO FOTÔMETRO SOLAR MULTIBANDA - FSM-4}

\subsubsection{Introdução}

Este instrumento portátil, desenvolvido para este trabalho, constitui-se um meio mais simples e econômico de coletar dados sobre a profundidade óptica de aerossóis ou vapor de água na atmosfera. É baseado na medição da intensidade do feixe solar, e, portanto, na transmitância atmosférica direta. A partir dessa transmitância, após a correção adequada do espalhamento e absorção por moléculas de gases, a profundidade óptica do aerossol ou o vapor de água pode ser obtido pelo ML e MLM.

A proposta de desenvolvimento de um fotômetro solar portátil e de baixo custo foi primeiramente apresentada por [27]. O uso de diodos emissores de luz na banda do infravermelho próximo mostra que esse tipo de componente poderia ser usado como alternativa para baratear o custo dos fotômetros [14]. O projeto do FSM-4, que é um fotômetro solar portátil de apontamento manual que opera em quatro bandas, visa não somente o baixo custo do equipamento, mas uma qualidade de dados aproximada dos fotômetros comerciais existentes.

Trata-se de um equipamento manual no apontamento solar, com precisão similar a equipamentos importados de sua categoria. O desenvolvimento do fotômetro partiu da necessidade de se obterem dados in situ para o estudo da profundidade óptica por aerossóis e coluna de água precipitável.

Como o uso objetivado do equipamento é monitorar a profundidade óptica dos aerossóis e água precipitável, foram escolhidas as faixas de $500 \mathrm{~nm}, 670 \mathrm{~nm}, 870 \mathrm{~nm}$ e $940 \mathrm{~nm}$, com banda passante de $10 \mathrm{~nm}$, usualmente empregada em fotômetros comerciais.

O projeto tomou por base os parâmetros normatizados pela $\mathrm{WMO} \mathrm{N}^{\circ} 08$ (2008)[28] para equipamentos usados nas medidas da radiação solar direta. Dentre esses parâmetros pode-se destacar o ângulo de abertura ou FOV (Field of View), tipo de elemento sensor, faixa de frequência e a banda passante dos seus filtros de interferência, para a radiação solar considerando um fotômetro solar comercial.

\subsubsection{Detalhes construtivos}

Em termos gerais, pode-se descrever o fotômetro dividindo o equipamento em quatro partes principais: tubo de colimação, filtro de interferência, elemento sensor e circuitos eletrônicos do amplificador de sinal e armazenamento de dados, conforme diagrama de blocos da Figura 3.3. 


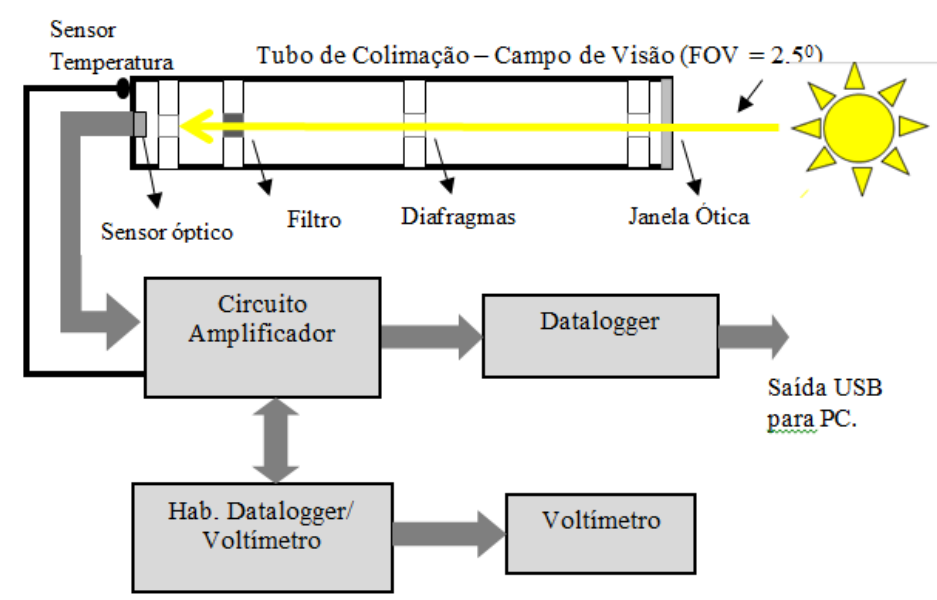

Figura 3.3. Diagrama de blocos do FSM-4.

Os tubos de colimação formam o corpo principal do fotômetro solar FSM-4. Essas características físicas são fundamentais para um equipamento óptico de forma a minimizar os efeitos de dilatação sobre essa estrutura principal do fotômetro. Para evitar a reflexões da radiação solar internas, no tubo de colimação, suas paredes internas e diafragmas são pintados com tinta preta fosca. Um importante acessório, o tripé, foi incorporado ao instrumento para facilitar seu apontamento para o sol.

O projeto do tubo de colimação da radiação solar obedece a parâmetros ditados pela WMO para equipamentos usados na medição da radiação solar direta, mostrado na Figura 3.4.

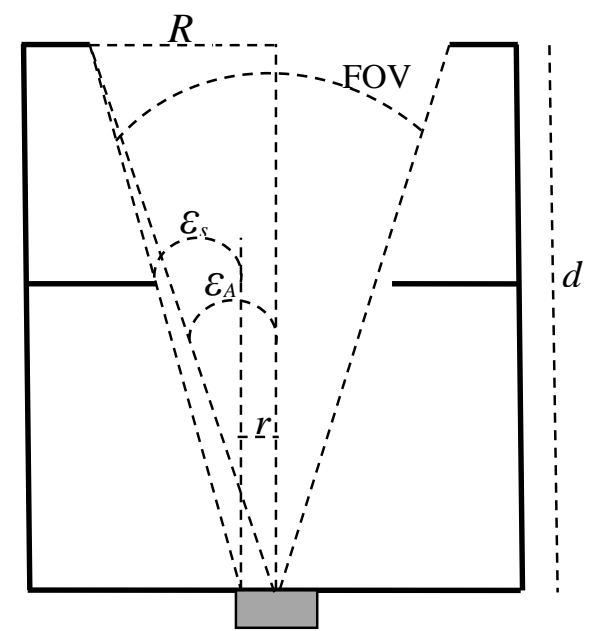

Figura 3.4. Configuração do tubo de colimação do FSM-4.

Para o FSM-4 os valores de $R$, $r$ e $d$, são: $2 \mathrm{~mm}, 1 \mathrm{~mm}$ e $90 \mathrm{~mm}$, respectivamente. Os ângulos de inclinação (slope angle) $\varepsilon_{s}$, e o meio ângulo de abertura $\varepsilon_{\mathrm{A}}$ são mostrados na Figura 3.4. Para o FSM-4 os ângulos $\varepsilon_{S}, \varepsilon_{A}$, são respectivamente, $0.63^{\circ}, 1.27^{\circ}$. O campo de visão do equipamento (FOV) é dado pela Equação 4.3, que mostra o ângulo máximo de $2.54^{\circ}$ graus para entrada da radiação solar direta, minimizando assim a entrada da radiação difusa [22].

Os filtros ópticos fabricados pela Andover Corporation - USA, os filtros ópticos são usados para limitar a chegada da radiação eletromagnética solar no sensor do fotômetro. Os filtros passabanda (band-pass) operam nas bandas de $500 \mathrm{~nm}, 670 \mathrm{~nm}, 870 \mathrm{~nm}$ e $940 \mathrm{~nm}$ com largura de banda 
de $10 \mathrm{~nm}$ e outras importantes características mostradas na Tabela 3.2. Esses componentes usados no FSM-4 possuem características semelhantes às bandas e largura de banda presentes nos filtros de interferência dos fotômetros CIMEL da rede AERONET.

Possuem coeficiente de variação da frequência central em função da temperatura dentro do range de $0.017 \mathrm{~nm} /{ }^{\circ} \mathrm{C}$ a $0.025 \mathrm{~nm} /{ }^{\circ} \mathrm{C}$ na faixa de 500 a $940 \mathrm{~nm}$, operando na temperatura de $+23^{\circ} \mathrm{C}$. Este coeficiente de variação de temperatura permite operar o equipamento entre $+10^{\circ} \mathrm{C}$ a $+35^{\circ} \mathrm{C}\left(\Delta \mathrm{t}=25^{\circ} \mathrm{C}\right)$ com variação da frequência central do filtro em somente $0.625 \mathrm{~nm}$. Uma janela óptica formada por uma lâmina de vidro de $1 \mathrm{~mm}$ de espessura na entrada do tubo de colimação permite a entrada da radiação eletromagnética solar. Esta tem a função de impedir a entrada de poeira e umidade no interior do equipamento, evitando assim danos ao filtro e ao elemento sensor. 
Tabela 3.2. Características dos filtros ópticos.

\begin{tabular}{ccccc}
\hline Filtro $-\mathbf{n m}$ & $\mathbf{5 0 0} \mathbf{~ n m}$ & $\mathbf{6 7 0} \mathbf{n m}$ & $\mathbf{8 7 0} \mathbf{n m}$ & $\mathbf{9 4 0} \mathbf{n m}$ \\
\hline Freq. Central $(\mathbf{n m})$ & 501.84 & 672.28 & $\mathbf{8 7 0 . 3 5}$ & 939.58 \\
\hline Larg. Banda $\mathbf{5 0 \%}(\mathbf{n m})$ & 10.70 & 9.88 & 10.01 & 8.54 \\
\hline Larg. Banda $\mathbf{1 0 \%}(\mathbf{n m})$ & 14.90 & 14.14 & 13.79 & 11.87 \\
\hline Larg. Banda 1\% (nm) & 21.67 & 20.73 & 20.00 & 29.00 \\
\hline Pico Transmissão (\%) & 66.72 & 69.84 & 61.80 & 60.96 \\
\hline Temp. $\left.^{\circ} \mathbf{C}\right)$ & 23.0 & 23.0 & 23.0 & 23.0 \\
\hline
\end{tabular}

O FSM-4 usa como elemento sensor um fotodiodo de silício. O fotodiodo converte a radiação eletromagnética do sol, ou seja, os fótons, em uma pequena voltagem em seus terminais. O fator de conversão varia em função da frequência da radiação solar, que é inversamente proporcional ao comprimento de onda. A Figura 3.5 mostra a curva de resposta desse fotodiodo (Curva de Resposta) para a relação (Amp./Watt) em função do comprimento de onda (wavelength) da radiação solar.

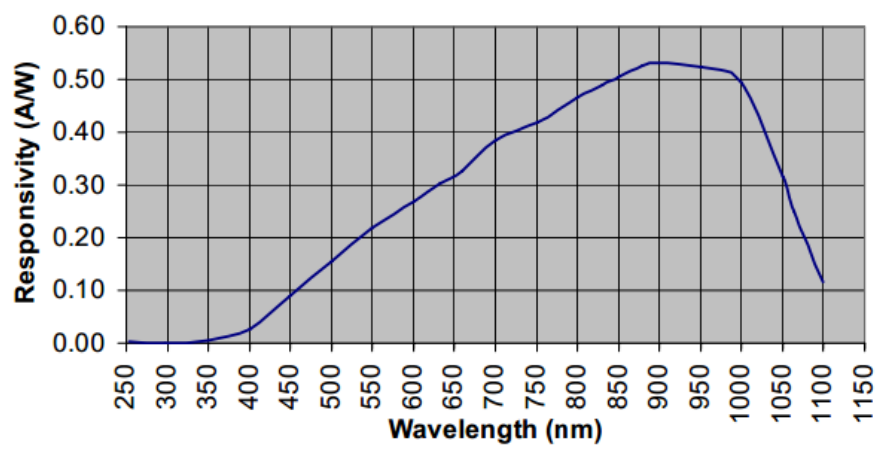

Figura 3.5. Curva de resposta do fotodiodo em função do comprimento de onda. (Fonte: Datasheet PDB-C139, Advanced Photonix, Inc.).

A Figura 3.6 a seguir mostra a aparência final do Fotômetro Solar Multibanda montado sobre um tripé.

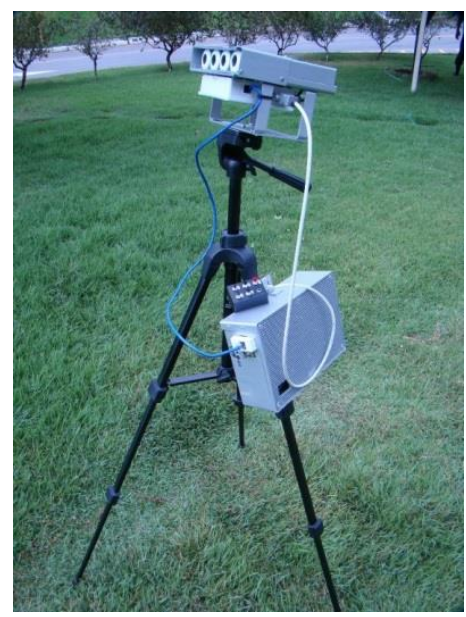

Figura 3.6. Fotômetro Solar - FSM-4 montado sobre tripé. 
Trata-se de um equipamento manual e de fácil operação. Após a montagem do equipamento em um tripé e conexão com o pequeno console através do conector do tipo "DB-9". O equipamento deve ser apontado para o sol e pressionado o botão "HAB" (habilita gravação de dado). Ao console pode ser conectado um voltímetro para monitoramento da tensão de saída do amplificador, por meio de quatro botões correspondentes aos quatro filtros do equipamento.

Para suporte ao processamento de dados coletados através do FSM-4, dois programas foram desenvolvidos na linguagem de programação "C" : SODDA - VALG (Software Depurador dados para Média entre valores máximos), que calcula a média dos valores máximos e SODDA MOP (Software Depurador de dados para Massa Óptica), que relaciona o valor máximo coletado com a massa óptica no horário da coleta.

\subsection{APLICAÇÃO DO MÉTODO DE LANGLEY MODIFICADO}

\subsubsection{Localização, condições meteorológicas do sítio e período de coleta.}

Foram feitas duas campanhas para obtenção dos dados com FSM-4 nesta região. A primeira campanha ocorreu entre 19 a 22 de Dezembro de 2012 e a segunda, entre os dias 4 a 7 de Setembro de 2013. O experimento foi realizado na cidade de Caicó que está localizada no semiárido brasileiro na posição $60^{\circ} 30^{\prime} 27^{\prime \prime} \mathrm{S}$ e $37^{\circ} 5^{\prime} 18^{\prime \prime} \mathrm{W}$ a uma altitude de $164 \mathrm{~m}$. A vegetação do semiárido brasileiro é formada pela caatinga que é uma vegetação endémica semelhante à savana, que é caracterizada por arbustos de pequeno porte, cactáceas e bromélias, adaptadas ao clima árido.

\section{Climatologia de Caicó}

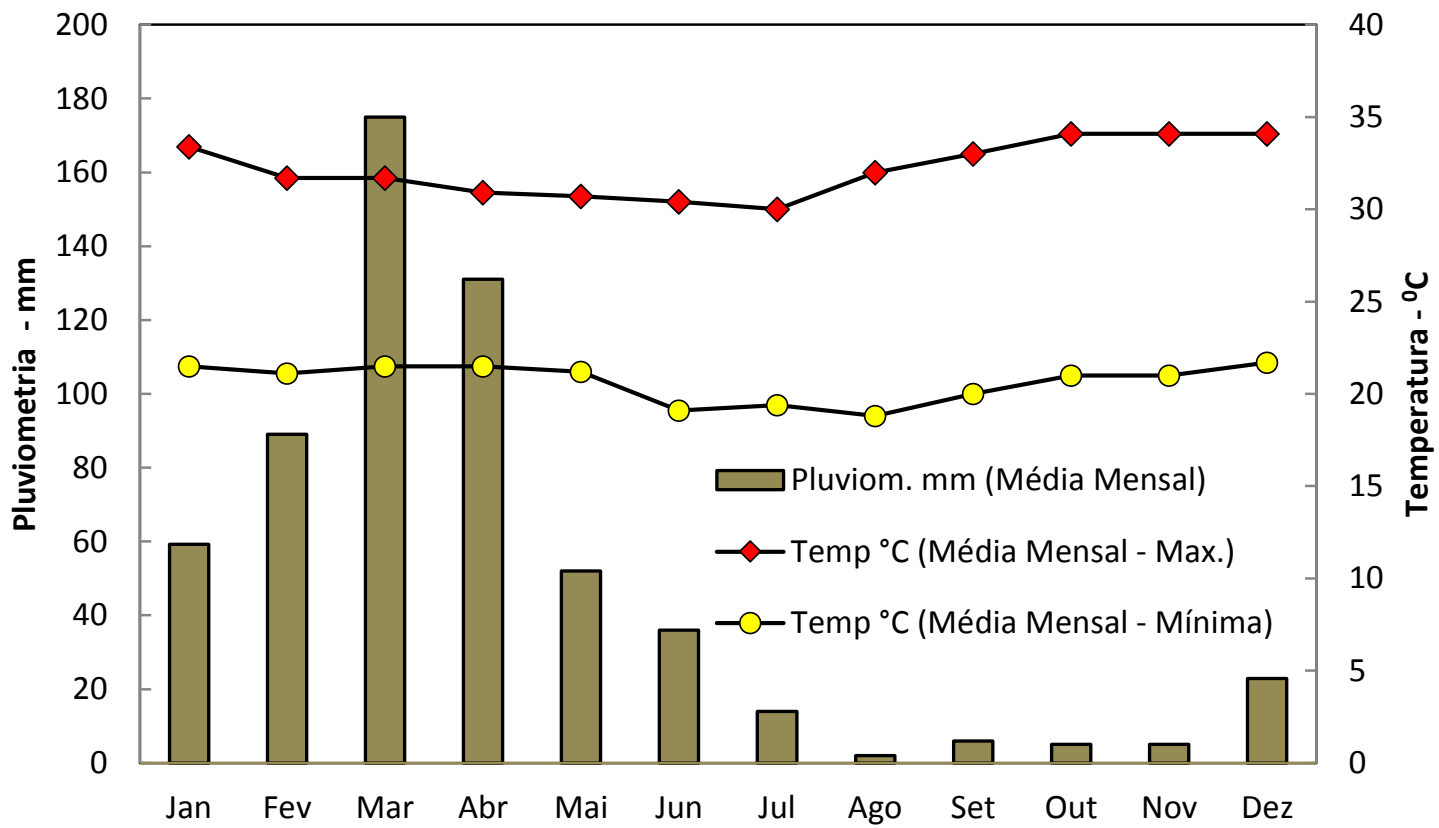

Figura 3.7. Climatologia anual em Caicó / Brasil. (Fonte: INMET).

A climatologia local não apresenta grandes variações de temperatura, umidade relativa do ar ou pressão de ar durante a estação seca, que vai de julho a dezembro, com pouca ou nenhuma chuva. Observa-se na figura 3.7 a pouca variabilidade dos valores de pluviometria entre os meses 
de agosto e dezembro no período seco indicam maior probabilidade de encontrar-se dias com poucas nuvens para campanhas feitas nesse período. Foram realizadas campanhas com duração de quatro dias, sendo uma em Dezembro de 2012 e outra em Setembro de 2013, que são em termos de climatologia o final e início do período mais seco. A temperatura é um fator climático que pode ter grande influência na variabilidade da $A O D$, devido as forças convectivas criadas na atmosfera. Apesar da pouca variabilidade entre as temperaturas máximas e mínimas, os altos valores de temperatura podem permitir a geração de grandes correntes convectivas na atmosfera que é o suficiente para causar instabilidade da AOD.

Os dados foram coletados no período da manhã, logo ao nascer do sol e no período da tarde, pouco antes do por do sol, ou seja, para dados coletados para massa óptica variando entre 12 e 2 , 5h30 - 7h15 hora local ( 08:30 e 10:15 am UT) e 15:40 - 17:30 hora local (18:40 - 20:30 UT). As coletas feitas com a massa óptica variando entre 2 e 5 são suficientes para uma boa calibração usando o ML [15].

As medições realizadas no período da manhã são melhores que as realizadas no período da tarde, quando as condições tornam-se favoráveis a um possível aumento de aerossóis trazidos por massas convectivas, provocada pelo aumento da temperatura do solo, [24]. A temperatura e as variações de umidade relativa durante os períodos de medições (manhã e tarde ), em dezembro de 2012 e setembro 2013 são mostrados na tabela 3.1. Em razão da indisponibilidade de parâmetros meteorológicos locais medidos em curto espaço de tempo ( $>1$ hora), a umidade relativa e temperatura foram consideradas constantes durante o experimento, ou seja, o valor considerado foi o obtido pela estação climatológica local às 7 hs (hora local) (10:00 - UT) e 15 hs (hora local) ( 18:00 - UT).

\section{RESULTADOS DO MLM NA BANDA DE 940 NM.}

A acentuada variação exponencial na banda de 940 nm, observada na Figura 4.1, mostra a forte dependência do vapor de água na atmosfera do sinal obtido com o FSM-4, comparada às outras curvas obtidas nas bandas estudadas.

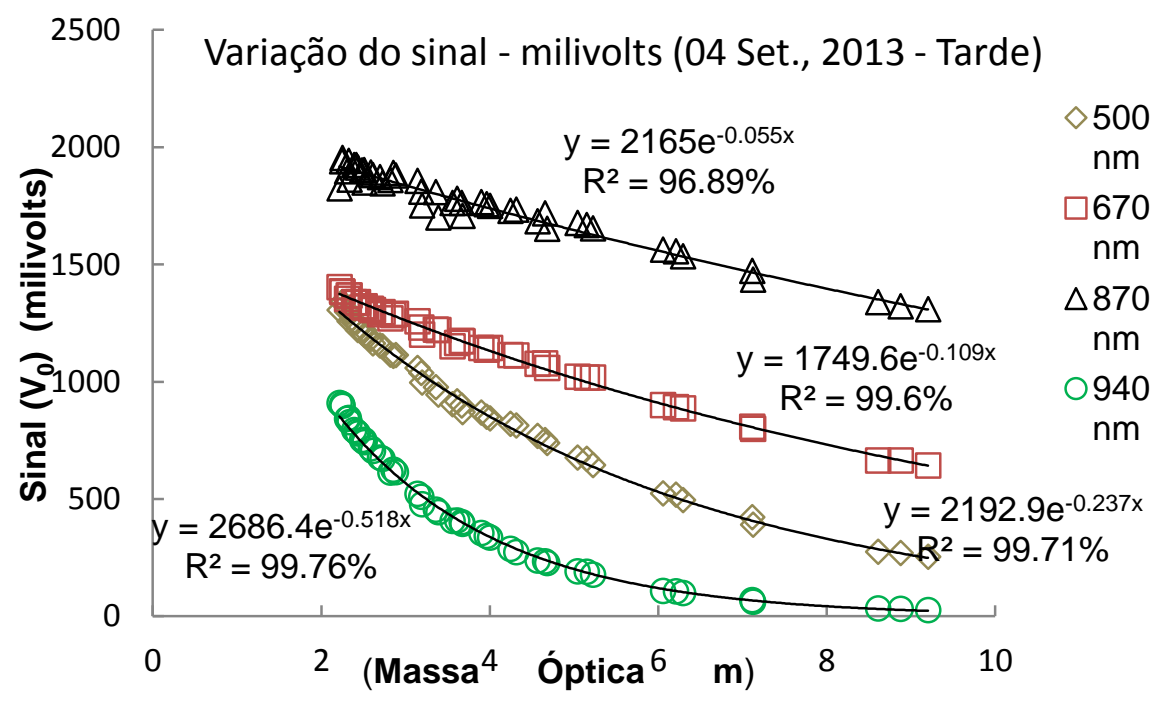

Figura 4.1. Variação exponencial dos dados coletados em função da massa óptica, com destaque para a acentuada variação exponencial na banda $940 \mathrm{~nm}$. 
Os dados coletados nas campanhas realizadas nos períodos de Dezembro de 2012 e Setembro de 2013 são apresentados na tabela 4.1. As retas ajustadas aos dados coletados apresentaram coeficientes de determinação muito forte para a maior parte dos meio dias de coleta, isto é, > 99\% nos períodos coletados, sendo o pior resultado obtido na manhã do dia 21 de dezembro com o $\mathrm{R}^{2}$ igual a $98.76 \%$.

A Figura 4.2 mostra como exemplo de reta ajustada aos dados obtidos na manhã do dia 04 de Dezembro, usando o MLM. Artigos sobre o assunto relatam que os dados coletados no período da manhã são melhores que os períodos da tarde devido uma possível elevação de aerossóis carreados por massa de ar convectivas.

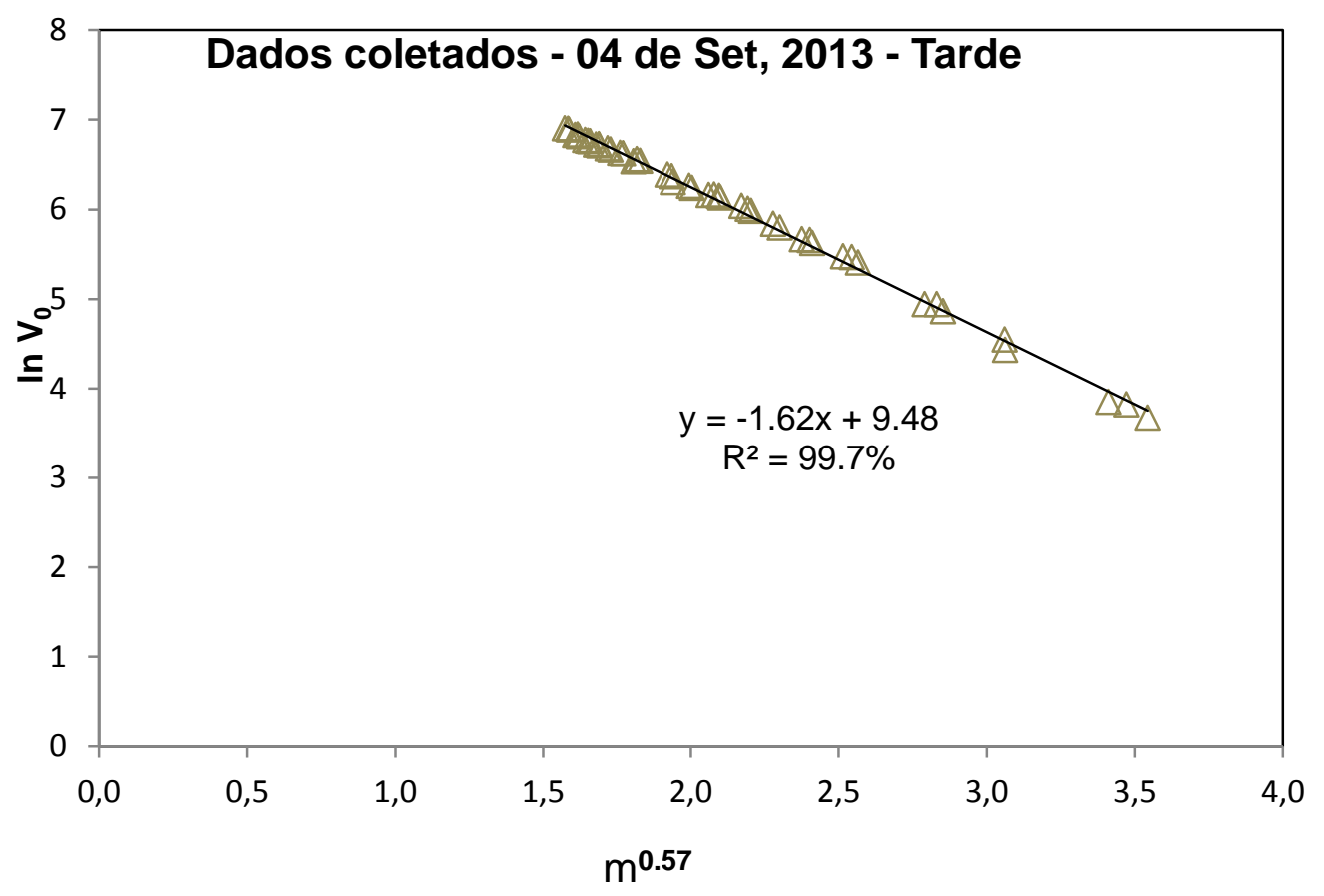

Figura 4.2. Exemplo de gráfico com dados linearizados para a banda de $940 \mathrm{~nm}$ obtidos na tarde de 04 de setembro de 2013.

Para a banda $940 \mathrm{~nm}$ foi notada uma estreita relação entre o aumento da umidade relativa e a variação da PWC, que em grande parte das medições realizadas, como mostra a Figura 4.3.

Tabela 4.1 - Valores observados em 940 nm em Dez., 2012 e Set., 2013.

\begin{tabular}{|c|c|c|c|c|}
\hline Data & $\begin{array}{c}\text { Equação ajustada } \\
-\mathbf{a x}+\mathbf{~ b}\end{array}$ & $\mathbf{R}^{\mathbf{2}} \mathbf{( \% )}$ & $\mathbf{a x} \mathbf{\pm} \mathbf{~} \mathbf{a}$ & $\mathbf{b} \pm \mathbf{\sigma b}$ \\
\hline 19, Dez - 2012 - Tarde & $-1.43 \mathrm{x}+9.96$ & 99.26 & $1.43 \pm 0.03$ & $9.96 \pm 0.06$ \\
\hline 20, Dez - 2012 - Manhã & $-1.47 x+9.626$ & 98.6 & $1.47 \pm 0.03$ & $9.92 \pm 0.06$ \\
\hline 20, Dez - 2012 - Tarde & $-1.41 x+9.55$ & 99.71 & $1.41 \pm 0.012$ & $9.55 \pm 0.03$ \\
\hline 21, Dez - 2012 - Manhã & $-1.65 x+9.83$ & 98.7 & $1.65 \pm 0.04$ & $9.83 \pm 0.1$ \\
\hline 21, Dez - 2012 - Tarde & $-1.45 x+9.73$ & 99.68 & $1.45 \pm 0.02$ & $9.73 \pm 0.04$ \\
\hline 22, Dez - 2012 - Manhã & $-1.66 x+9.88$ & 98.82 & $1.66 \pm 0.03$ & $9.88 \pm 0.08$ \\
\hline 04, Set., 2013 - Tarde & $-1.62 x+9.48$ & 99.7 & $1.62 \pm 0.015$ & $9.48 \pm 0.033$ \\
\hline 05, Set., 2013 - Manhã & $-1.64 x+9.71$ & 98.87 & $1.64 \pm 0.03$ & $9.71 \pm 0.08$ \\
\hline 05, Set., 2013 - Tarde & $-1.31 x+9.2$ & 99.5 & $1.31 \pm 0.02$ & $9.2 \pm 0.04$ \\
\hline 06, Set., 2013 - Manhã & $-1.43 x+9.56$ & 99.69 & $1.43 \pm 0.02$ & $9.56 \pm 0.03$ \\
\hline 07, Set., 2013 - Manhã & $-1.46 x+9.60$ & 99.45 & $1.46 \pm 0.025$ & $9.6 \pm 0.05$ \\
\hline
\end{tabular}


Os demais dados observados para a banda de $940 \mathrm{~nm}$ são mostrados na tabela 4.1, para os períodos da tarde e manhã. A tabela 3.2 apresenta os coeficientes lineares e angulares das retas ajustadas ao dados coletados. São também apresentadas suas variabilidades dadas por oa e ob.

Os coeficientes de determinação $\left(R^{2}\right)$ mostram o ótimo ajuste dos dados coletados às curvas ajustadas, mas não são determinantes na indicação de bons resultados. Somente a análise da variabilidade do coeficiente linear da reta ajustada (a) poderá mostrar se a constante de calibração foi encontrada, visto na tabela 4.3.

A tabela 4.2 mostra a forte correlação entre a umidade relativa e $W$ que é dado em centrímetros $(\mathrm{cm})$. $O$ calculo do $W$ é feito usando o coeficiente angular $\left(T_{w}\right)$ da reta ajustada, aplicando-se os coeficientes " $a$ " e " $b$ " do filtro [2].

Tabela 4.2. A Coluna de água precipitável, dado na fómula por W (valor médio \pm Desvio padrão amostral) e compara à umidade relativa (UR).

\begin{tabular}{|c|c|c|c|}
\hline Data & $a W^{b}$ & $\begin{array}{l}W \text { “cm" (p/ } a=0.69 \text { e } b=0.57) \\
\text { Valor Médio } \mathrm{p} / \text { período coleta }\end{array}$ & UR (\%) \\
\hline 19, Dez - 2012 - Tarde & $1.43 \pm 0.03$ & $3.6 \pm 0.17$ & 29 \\
\hline 20, Dez - 2012 - Manhã & $1.47 \pm 0.03$ & $3.8 \pm 0.17$ & 55 \\
\hline 20, Dez - 2012 - Tarde & $1.41 \pm 0.012$ & $3.5 \pm 0.06$ & 26 \\
\hline 21, Dez - 2012 - Manhã & $1.65 \pm 0.04$ & $4.6 \pm 0.24$ & 53 \\
\hline 21, Dez - 2012 - Tarde & $1.45 \pm 0.02$ & $3.7 \pm 0.11$ & 27 \\
\hline 22, Dez - 2012 - Manhã & $1.66 \pm 0.03$ & $4.7 \pm 0.18$ & 52 \\
\hline 04, Set., 2013 - Tarde & $1.62 \pm 0.015$ & $4.5 \pm 0.08$ & 37 \\
\hline 05, Set., 2013 - Manhã & $1.64 \pm 0.03$ & $4.6 \pm 0.18$ & 57 \\
\hline 05, Set., 2013 - Tarde & $1.31 \pm 0.02$ & $3.1 \pm 0.10$ & 37 \\
\hline 06, Set., 2013 - Manhã & $1.43 \pm 0.02$ & $3.6 \pm 0.10$ & 56 \\
\hline 07, Set., 2013 - Manhã & $1.46 \pm 0.025$ & $3.7 \pm 0.14$ & 57 \\
\hline
\end{tabular}

Umidade Relativa x Coluna de Vapor de Água (PWC)

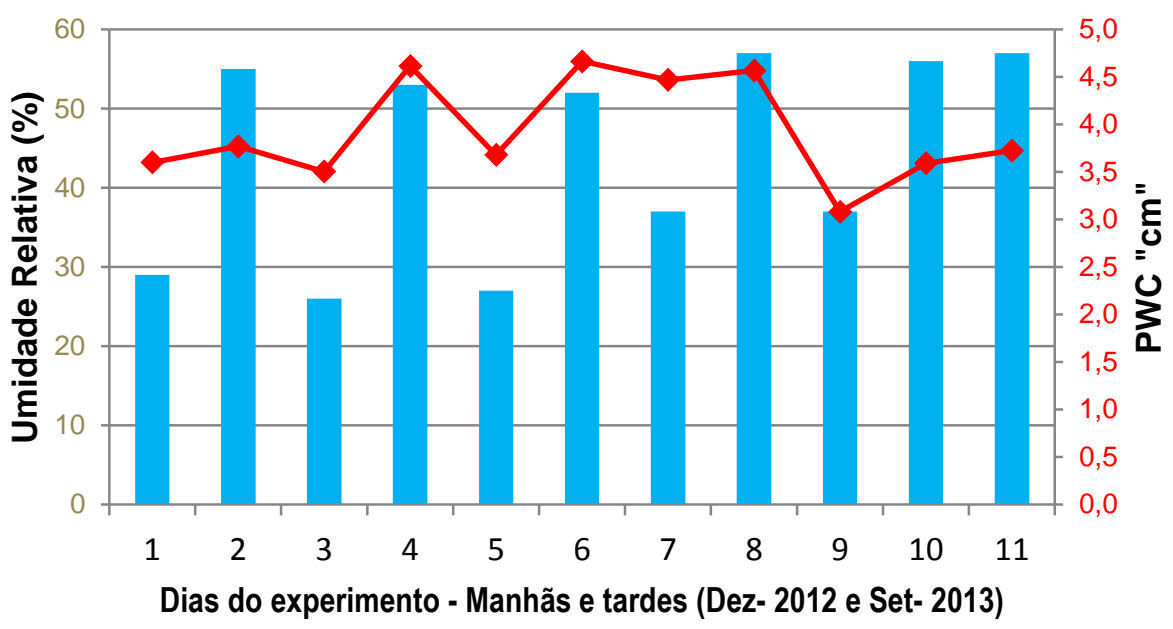

Figura. 4.3. Gráfico mostrando correlação entre o PWC x RH(\%) para Caicó.

A Figura 4.3 mostra de forma gráfica os dados da tabela 4.2. Nota-se que, a variação do PWC nos meio dias (manhãs e tardes) acompanha a variação da umidade relativa média para as manhãs e tardes no sítio estudado. Nota-se que os valores de PWC acompanham a variação da umidade relativa em aproximadamente $90 \%$ dos horários de medidas. 
A umidade relativa serve apenas como parâmetro comparativo, pois, como essa medida é feita ao nível do solo seus valores podem não corresponder integralmente ao valor da coluna de vapor de água precipitável. A PWC que leva em conta toda água existente na coluna, e dependendo da região e período do ano a coluna pode extender-se até à estratosfera, conforme dados fornecidos por sondas meteorológicas.

Tabela 4.3. Valores de $V_{0}$ obtidos para a banda de $940 \mathrm{~nm}$.

\begin{tabular}{|c|c|c|}
\hline Data & In $\boldsymbol{V}_{\mathbf{0}}$ & $\boldsymbol{V}_{\mathbf{0}}$ \\
\hline 19, Dez - 2012 - Tarde & 9.96 & 21162.8 \\
\hline 20, Dez - 2012 - Manhã & 9.92 & 20332.99 \\
\hline 20, Dez - 2012 - Tarde & 9.55 & 14044.69 \\
\hline 21, Dez - 2012 - Manhã & 9.83 & 18582.95 \\
\hline 21, Dez - 2012 - Tarde & 9.73 & 16814.55 \\
\hline 22, Dez - 2012 - Manhã & 9.88 & 19535.72 \\
\hline 04, Set., 2013 - Tarde & 9.48 & 13095.19 \\
\hline 05, Set., 2013 - Manhã & 9.71 & 16481.6 \\
\hline 05, Set., 2013 - Tarde & 9.20 & 9897.129 \\
\hline 06, Set., 2013 - Manhã & 9.56 & 14185.85 \\
\hline 07, Set., 2013 - Manhã & 9.60 & 14764.78 \\
\hline
\end{tabular}

Os coeficientes lineares das retas $\left(V_{0}\right)$, obtidas nas duas campanhas, por meio do MLM, são apresentados na tabela 4.3, no qual pode-se observa-se uma alta variabilidade desses valores. Aplicando-se a combinação dos onze valores de $V_{0}$, dois a dois, por meio da equação 4.1 , obtemse cinquenta valores.

$$
C_{2}^{11}=\frac{11 !}{2 !(11-2) !}=55
$$

A combinação dos valores de $V_{0}$ são calculados por meio da equação 4.2 [9]:

$$
\Delta V_{o} / V_{o}=\left|\frac{\left(V_{o n}-V_{o(n+1)}\right)}{V_{O(n+1)}}\right|,
$$

De forma similar ao $M L$, o MLM exige que a constante $V_{0}$ seja repetida durante vários dias, porém com desvio padrão amostral um pouco maior que na banda visível, ou seja, SD $\leq 3 \%$. Devido esse maior SD pode-se aceitar um desvio percentual entre os valores de $V_{0}$ de $4 \%$, que é um pouco maior que a banda visível, para uma avaliação inicial da dispersão dos valores, que é mostrado na Figura 4.4. Foram encontrados somente cinco combinações de $\Delta V_{0} / V_{0}$ com resultados aceitáveis, ou seja, com variação menor que $4 \%$ para esse teste inicial, portanto, insuficiente para a aplicação da metodologia.

A alta dispersão dos valores de $V_{0}$ indica que o sítio estudo apresentou a requerida estabilidade da profundidade óptica devido o vapor de água. Mesmo no curto período de coleta de dados exigido para o MLM, ou seja, aproximadamente 1:30 hs no início da manhã ou final da tarde, não houve a requerida estabilidade. 


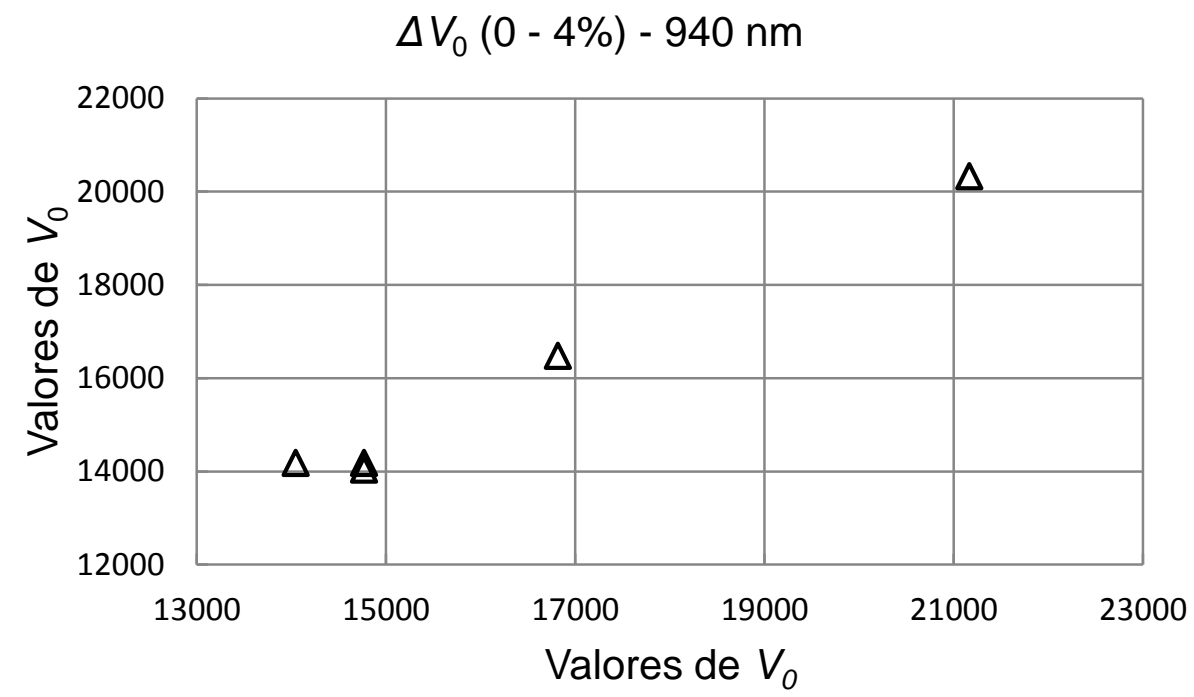

Figura 4.4. Dispersão totasl entre os valores de V0 comparado com os pares de V0 ( $\Delta \mathrm{V} 0)$ com variação entre 0 a $4 \%$.

\section{CONCLUSÕES}

A variabilidade dos coeficientes $V_{0}$ para o MLM nesse experimento indicam que o sítio em Caicó não apresentou a almejada estabilidade para a aplicação do método modificado de Langley. A divergência dos valores absolutos mostram uma grande divergência percentual entre os valores de $V_{0}$, superiores às diferenças percentuais obtidas nas bandas de $500 \mathrm{~nm}, 670 \mathrm{~nm}$ e $870 \mathrm{~nm}$. Isto mostra uma maior exigência da condições de estabilidade atmosférica, necessária para a calibração do fotômetro, na banda em que o vapor de água sofre forte absorção.

O reduzido tempo de campanha também colaborou com para a não obtenção de resultados mais significativos. Somente com campanhas mais prolongadas pode-se afimar com mais certeza que o sítio em Caicó não reune as condições necessárias. A variabilidade da umidade relativa presente no sítio pode ser considerada como um forte indicativo para esta instabilidade da PWC no período estudado. Somente com levantamentos realizados em outros períodos, semelhantes aos executados em setembro e dezembro, o sítio poderá ser descartado quanto a aplicação do MLM.

\section{REFERÊNCIAS}

1. Berk, A., L.S. Bernstein, and D.C. Robertson, MODTRAN: A Moderate Resolution Model for LOWTRAN7, technical report, Geophys. Dir., Phillips Lab., Hanscom Air Force Base, Mass., 1989.

2. Bokoye, A. I., Royer, A., Santer, R., Calibration of sun radiometer-Based Atmospheric Water vapour Retrievals using GPS Meteorology, Journal of Atmospheric and oceanic technology, DOI: 10.1175/JTECH2011.1, 2006.

3. Bruegge, C.J., J.E. Conel, R.O. Green, J.S. Margolis, R.G. Holm, and G. Toon, Water vapor column abundance retrievals during FIFE, J. Geophys. Res., 97, 18,759-18,768, 1992 
4. Castanho, A. D. A., Propriedades ópticas das partículas de aerossol e uma nova tecnologia para obtenção de profundidade óptica via satélite sobre São Paulo, Tese de Doutorado, Instituto de Física, USP, 2005

5. Gatebe, C. K., O. Dubovik, O., King, M. D., Sinyuk, A., Simultaneous retrieval of aerosol and surface optical properties from combined airborne- and ground-based direct and diffuse radiometric measurements, Atmos. Chem. Phys., 10, 2777-2794, 2010.

6. Halthore, R. N., Eck, T. F., Holben, B. N., Markhan, B. L.; Sunphotometric measurements of atmospheric water vapor column abundance in the $940 \mathrm{~nm}$ band, Journal of Geophysical Research, vol 102, No D4, pages 4343-4352, February 27, 1992.

7. Harrison, L., Michalsky, J.; Objective algorithms for the retrieval of optical depths from groundbased measurements, APPLIED OPTICS Vol. 33, No. 22, pp 5126-5132, 1 August 1994.

8. Ingold, T., B. Schmid, C. Mätzler, P. Demoulin, and N. Kämpfer,: Modeled and empirical approaches for retrieving columnar water vapor from solar transmittance measurements in the 0.72, 0.82, and 0.94 m absorption bands. J. Geophys. Res., 105 (D19), 24 327-24 344, 2000.

9. Kaufman, Y.J.; Fraser, R. S.; Light extinction by aerosols during summer pollution.; J. Climate Appl. Meteor.; V.22, 1694-1706, October,1983.

10. Kneizys, F.X., Shettle, E.P., Chetwynd, J.H., Abreu, L.W., Anderson, G.P., Gallery, W.0, Selby, J.E.A., Clough, S.A.., Users Guide to LOWTRAN 7, AFGL-TR-88-O 177, 1988.

11. Liou, K. N., An Introduction to Atmospheric Radiation, Academic Press, Second Edition, 579p, 2002.

12. Liu, C., Li, Y., Gao, W., Shi, R., Bai, k.,; Retrieval of columnar water vapor using measurements over northern China, Journal of Applied Remote Sensing Vol. 5, 2011.

13. Michalsky, J. J.; Liljegren, J. C.; Harrison, L. C.; A comparison of Sun photometer derivations of total column water vapor and ozone to standard measures of same at the Suthern Great Plains Atmospheric Radiation Measurement site., Journal of Geophysical Research, vol. 100, N0. D12, pages 25,995 - 26,003, Dec 20, 1995.

14. Mims, F. M., III,: An inexpensive and stable LED Sun photometer for measuring the water vapor column over South Texas from 1990 to 2001. Geophys. Res. Lett., 29, 1642, doi:10.1029/ 2002GL014776, 2002.

15. Porter, J. N.; Miller, M; Pietras, C., and Motell, C.: Ship-based Sun photometer measurements using Microtops Sun photometers, J. Atmos. Ocean. Tech., 18, 765-774, 2001.

16. Randall, D.A., R.A. Wood, S. Bony, R. Colman, T. Fichefet, J. Fyfe, V. Kattsov, A. Pitman, J. Shukla, J. Srinivasan, R.J. Stouffer, A. Sumi and K.E. Taylor: Climate Models and Their Evaluation. In: Climate Change 2007: The Physical Science Basis. Contribution of Working Group I to the Fourth Assessment Report of the Intergovernmental Panel on Climate Change, 2007.

17. Reagan, J. A., Thome, K., Herman, B., Gall, R., Water vapor measurement in 0.94micro absorption band: Calibration, measurements, and data applications. Proc. International Geoscience and Remote Sensing Symp.' 87 Symposium, University of Michigan, Ann Arbor, 6357, (1987)b.

18. Reagan, J. A.; Thome, K. J.; Herman, B. M.; A Simple Instrument and Technique for Measuring 
Columnar Water Vapor via Near-IR Differencial Solar Transmission Measurements, IEEE Transactions on Geoscience and Remote Sensing, Vol. 30, NO. 4, July, 1992.

19. Schmid, B.; Thome, K. J.; Demoulin, P.; Peter, R.; Matzler, C.; Sekler, J.; Comparison of modeled and empirical approaches for retrieving columnar water vapor from solar transmittance measurements in the $0.94 \mu \mathrm{m}$ region., Journal of Geophysical Research, vol. 101, N0. D5, pages 9345-9358, april, 27 - 1996.

20. Schmid, B., Michalsky, J. J., Slater, D. W., Barnard, J; C., Halthore, R. N., J. C. Liljegren, J. C., Holben, B. N., Eck,T. F., Livingston, J. M., Russell, P. B.; Comparison of columnar water-vapor measurements from solar transmittance methods., Appl. Opt. 40(12), 1886-1896, 2001.

21. Shiobara, M., Spinhirne, J. D., Uchiyama, A., and Asano, S., 1991, Optical depth measurements of aerosol, cloud and water vapor using sun photometers during the FIRE Cirrus IFO II. Journal of Applied Meteorology, 35, 36-46.

22. Slusser, J., Gibson, J., Biglow, D., Langley Method of Calibrating UV filter Radiometers, Journal of Geophysical Research, vol. 105, pg 4841 - 4849 - February 27- 2000.

23. Smirnov, A.; Holben, B. N.; Eck, T. F.; Dubovik, O.; Slutsker, I.; Cloud-Screening and Quality Control Algorithms for the AERONET Database, Remote Sense Invironment, 2000.

24. Shaw, G. E., Sun Photometry, Bull. Amer. Meteor. Soc., 64:4-11, 1983.

25. Taha, G., Box, G. P.; Water vapor column abundance retrieval using multispectral radiometer measurements in Sydney, Australia, Australian Met. Magazine 51, 165-172, 2002.

26. Thomason, L. W., Extinction of near infrared solar radiation as a means for remote determination of atmospheric water vapor. Ph.D. Thesis. University of Arizona, Tucson, Arizona, 122 pp, (1985).

27. Volz, F.E., Economical multispectral sun photometer for measurements of aerosol extinction

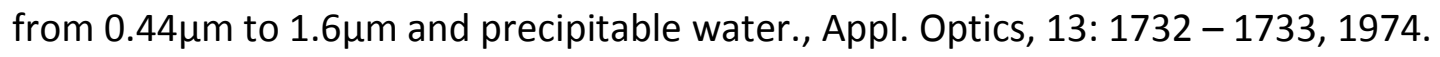

28. WMO - Guide to Meteorological Instruments and Methods of Observation - WMO No.8 Seventh edition - 2008. 